\title{
Ectopic Human Fasciola hepatica Infection by an Adult Worm in the Mesocolon
}

\author{
Ah Jin Kim', Chang Hwan Choi², Sun Keun Choi³, Yong Woon Shin', Yun-Kyu Park ${ }^{5}$, Lucia Kim², Suk Jin Choi², \\ Jee Young Han², Joon Mee Kim², Young Chae Chu², In Suh Park ${ }^{2, *}$ \\ 'Department of Emergency Medicine, Inha University Hospital, Inha University School of Medicine, Incheon 22332, Korea; ${ }^{2}$ Department of \\ Pathology, Inha University Hospital, Inha University School of Medicine, Incheon 22332, Korea; ${ }^{3}$ Department of Surgery, Inha University Hospital, \\ Inha University School of Medicine, Incheon 22332, Korea; ${ }^{4}$ Department of Internal Medicine, Inha University Hospital, Inha University School of \\ Medicine, Incheon 22332, Korea; 5 Department of Parasitology, Inha University School of Medicine, Incheon 22332, Korea
}

\begin{abstract}
We report here an ectopic case of Fasciola hepatica infection confirmed by recovery of an adult worm in the mesocolon. A 56-year-old female was admitted to our hospital with discomfort and pain in the left lower quadrant of the abdomen. Abdominal CT showed 3 abscesses in the left upper quadrant, mesentery, and pelvic cavity. On surgical exploration, abscess pockets were found in the mesocolon of the sigmoid colon and transverse colon. A leaf-like worm found in the abscess pocket of the mesocolon of the left colon was diagnosed as an adult fluke of $F$. hepatica. Histologically, numerous eggs of $F$. hepatica were noted with acute and chronic granulomatous inflammations in the subserosa and pericolic adipose tissues. Conclusively, a rare case of ectopic fascioliasis has been confirmed in this study by the adult worm recovery of $F$. hepatica in the mesocolon.
\end{abstract}

Key words: Fasciola hepatica, ectopic fascioliasis, human, mesocolon

\section{INTRODUCTION}

Fasciolid trematodes, such as Fasciola hepatica and Fasciola gigantica, are primarily the liver flukes of herbivorous mammals, i.e., sheep, cattle, and goats. They accidentally infect humans. This trematode infection, i.e., fascioliasis, is widely distributed throughout the world $[1,2]$. In particular, many cases have been reported from South America, Northern Africa, Iran, and Western Europe [1-3]. Recently, hyperendemic areas were reported in South America and Egypt [4-6]. Although several human cases have been sporadically reported in the Republic of Korea, endemic areas have not been known yet [7-11].

In human fascioliasis, fasciolid worms are commonly found in the bile duct and gall bladder. However, they are sometimes detected in ectopic foci, i.e., cecum, ascending colon, brain, eye, spinal cord, subcutaneous tissues, and lymph nodes [7$9,12-16]$. Neurological and ocular fascioliasis in humans was reported to be fatal or disabling [17]. Intestinal perforation

- Received 17 April 2015, revised 18 November 2015, accepted 21 November 2015. *Corresponding author (ispap@inha.ac.kr) (c) 2015, Korean Society for Parasitology and Tropical Medicine This is an Open Access article distributed under the terms of the Creative Commons Attribution Non-Commercial License (http://creativecommons.org/licenses/by-nc/3.0) which permits unrestricted non-commercial use, distribution, and reproduction in any medium, provided the original work is properly cited. was seen in dead rabbits experimentally infected with $F$. hepati$\mathrm{ca}$, and fascioliasis could be fatal due to various complications [18]. Ectopic fascioliasis in various organs such as the pancreas, cecum, eye, and subcutaneous tissues have been reported in Korea [7,19-21].

Fasciola infection induces histopathologic changes where there are parasitic lesions. The histopathologic findings in ectopic fascioliasis are characterized by eosinophilic infiltration and granulomatous lesions formed around eggs and/or worms. Inflammation and fibrosis are seen in the migratory track of the worms. The chronic granulomatous lesion lacks lymphocytes and giant cells $[7,12,16,18]$.

We report a case of human ectopic fascioliasis in which an adult fluke and eggs were found in the mesocolon in a surgically resected specimen. The patient consumed fresh water plants. Mesocolon abscess by ectopic fascioliasis should be given a thought in the differential diagnosis of patients exposed to fresh water plants.

\section{CASE RECORD}

A 56-year-old female was admitted to a local hospital with discomfort and pain in the left lower quadrant of the abdomen for 10 days. The patient had a surgical history of appen- 

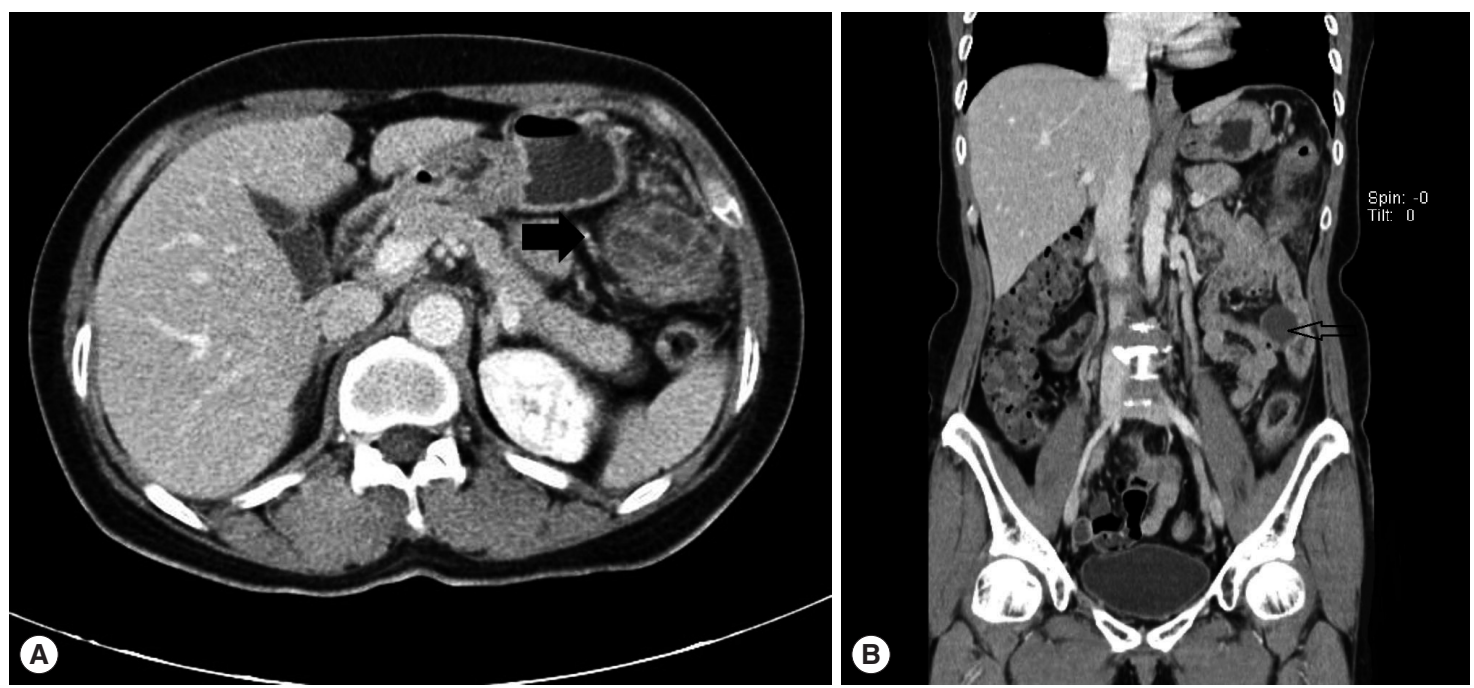

Fig. 1. (A) Abdominal CT showing 2 abscesses in the anterior aspect of the distal transverse colon (4 cm, arrow) in the interbowel space of the left middle abdomen $(3.5 \mathrm{~cm})$. (B) The coronal view of (A). The arrow indicates the abscess of the interbowel space of the left middle abdomen.
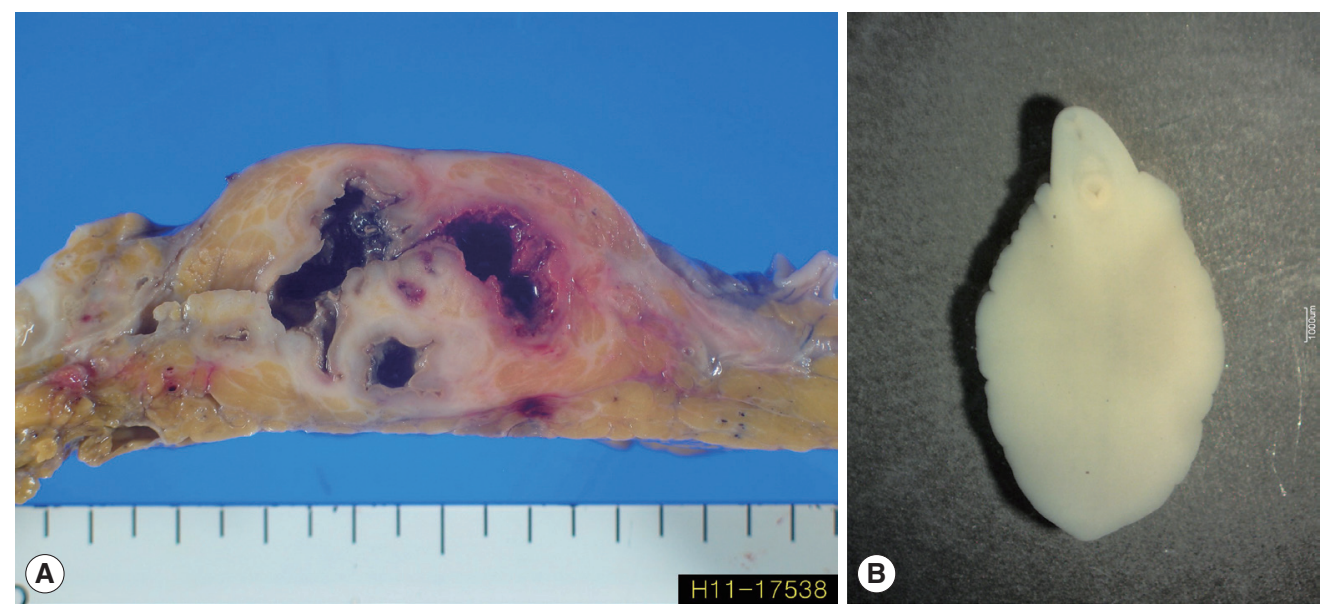

Fig. 2. (A) The abscess pocket in the mesocolon of the sigmoid colon showing a multiloculated appearance with inflamed dense fibrous tissue. The cavity contains dark-brownish fluid. (B) The adult fluke of $F$. hepatica found in the abscess cavity of the mesocolon which measured $1.3 \mathrm{~cm}$ in body length and $0.4 \mathrm{~cm}$ in body width.

dectomy 4 years ago. She had never traveled abroad. She had taken extracts from lotus leaf and mixed plants for a few months.

She was admitted after the symptom onset 10 days later (21 October 2011); an abscess was found in the left lower paracolic gutter by abdominal CT and the patient was treated with oral antibiotics. Twenty days after the first CT, on a follow-up CT, 2 additional abscesses were found at the splenic flexure in the left upper quadrant area and the pelvic cavity. Her symptoms did not improve despite the antibiotic therapy.

She was transferred to our hospital on 10 November 2011.
At that time, physical examination revealed tenderness in the left lower quadrant of the abdomen. A blood test showed eosinophilia. WBC count was $8,200 / \mu l$, and the proportion of eosinophils was $13.8 \%$. CRP was $7.41 \mathrm{mg} / \mathrm{dl}$, and serum electrolytes and liver enzymes were within normal range. An abdominal CT showed 3 abscesses in the left upper quadrant, mesentery, and pelvic cavity. On the 11th hospital day after transfer (21 November 2011), a 4th abdominal CT was performed. Two abscesses were found in the anterior aspect of the distal transverse colon $(4 \mathrm{~cm})$ and the interbowel space of the left mid abdomen $(3.5 \mathrm{~cm})$ (Fig. 1A, B). The abscess in the 


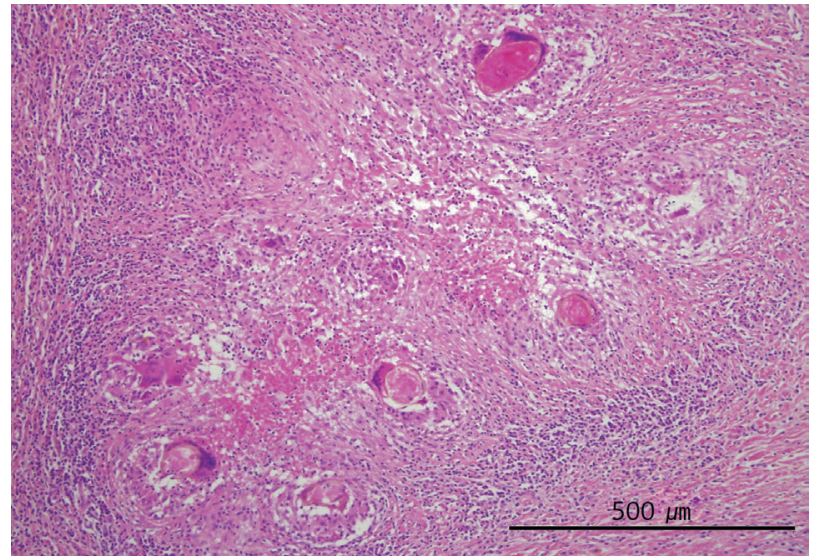

Fig. 3. Numerous eggs of $F$. hepatica found within acute and chronic granulomatous inflammations in the subserosa of the sigmoid colon. Eosinophils show diffuse infiltrations with edematous changes in the subserosa. H\&E stain, $\times 100$.

pelvic cavity had disappeared. Small bowel series and colonoscopy showed no remarkable findings except a diverticulum at the duodenal third portion.

A parasitic infection was suspected because of eosinophilia and radiologic findings. No larvae or ova of parasites were detected in the stool examination. Routine parasitic ELISA test (Architect i2000SR, Abbott Diagnostics, Abott Park, Illinois, USA) was requested, and the result was positive for cysticercus cellulosae and sparganum.

On the 18th hospital day after transfer (28 November 2011), the patient underwent transverse and left colectomy and anterior resection under the impression of cysticercosis. Surgical exploration showed abscess pockets in the mesocolon of the sigmoid colon and distal one third of the transverse colon (Fig. 2A). The leaf-like worm was found in the abscess pocket of the mesocolon of the left colon. The cavity of the abscess contained dark-brownish fluid. The leaflet-like worm was diagnosed as an adult fluke of $F$. hepatica (Fig. 2B). The adult fluke of $F$. hepatica measured $1.3 \mathrm{~cm}$ in body length and $0.4 \mathrm{~cm}$ in body width. The worm had 2 suckers (oral and ventral) and a characteristic conical projection.

Pathological examinations were performed for the resected colon. Histologically, numerous eggs of $F$. hepatica were noted with acute and chronic granulomatous inflammations in the subserosa and pericolic adipose tissues of the sigmoid colon and descending colon. Necrotic cysts and abscess formations were observed around the granulomas. Eosinophils showed diffuse infiltrations in the subserosa, and scattered eosinophils

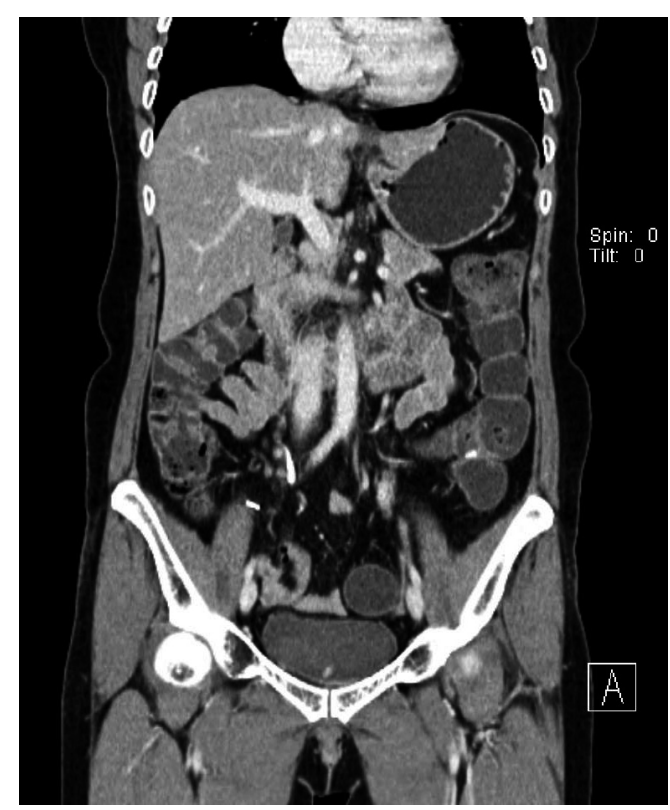

Fig. 4. No abnormal lesion was observed after 1-year follow-up.

were noted in the submucosa with edematous changes (Fig. 3). After the operation, ELISA for $F$. hepatica was positive.

She was discharged on the 5th postoperative day without additional chemotherapy for parasites. A year later, she did not complain of any symptoms related to the disease, and an abdominal CT finding was normal except the left hemicolectomy (Fig. 4).

\section{DISCUSSION}

In extrabiliary fascioliasis, juvenile or larval worms are found in most cases but adult flukes are rarely detected. In the present study, it is interesting that eggs and adult flukes were found at the same time in the extrabiliary mesocolon.

F. hepatica infection is a zoonosis which can infest humans and other mammals. Humans are an incidental host, but human fascioliasis occurs worldwide. The Republic of Korea is not an endemic area of human fascioliasis [2].

This patient is presumed to have been infected by eating lotus extracts. Snails are known to be the first intermediate host. In Korea, the prevalence of $F$. hepatica-infected snails was estimated to be 3.4\% [22]. Water lotus grows or is grown in wetlands or ponds. This habitat is similar to the snail habitat. Lotus extract is made from grinding lotus leaf or cutting lotus stem. We think that the metacercariae of $F$. hepatica are stuck to the lotus, and raw lotus extract could infect humans. 
The parasite penetrates the intestinal wall to reach the peritoneum. After a few days, F. hepatica penetrates the capsule of the liver. Through the liver parenchyma, the juvenile fluke reaches the bile duct, where it matures into an adult and eggs are passed [1]. The adult fluke in this case may have been growing in the mesocolon.

The usual forms of human fascioliasis are masses or abscesses in the liver parenchyma, or masses in the bile duct. Related clinical manifestations include abdominal pain, fever, hepatomegaly, urticaria, and so on. These symptoms occur in the acute phase of fascioliasis. Jaundice, cholecystitis, or cholestasis may be seen in the chronic phase. Ectopic sites, except in the hepatobiliary system, are not so rare, and various sites have been reported, including the cecum, ascending colon, brain, eye, spine, subcutaneous tissues, and cervical and inguinal lymph nodes [7-9,12-16]. In the first reported case of ectopic fascioliasis in Korea, a juvenile form of a Fasciola species worm was found in the cecum and ascending colon in 1982 [7].

The ascending colon and cecum are located near the liver, which mean the usual routes toward the liver. Our case is a rare occasion and more meaningful because abscesses, tracts, eggs, and adult flukes were found in the transverse colon, descending colon, or mesocolon.

Almost all fascioliasis cases occur by direct penetration through the bowel wall, and only a few cases of infection occur through lymphatic or blood circulation $[15,16,23]$. In our case, numerous eggs, granulomas, and abscesses were found in the fibroadipose tissue of the mesocolon. No mucosal lesion or orifice within the cavity contained $F$. hepatica, and there was no evidence of bodies or eggs of $F$. hepatica in blood vessels, lymphatic vessels, or lymph nodes. We conclude that the parasite developed in the transverse colon and descending colon by direct penetration rather than through lymphatics or blood circulation.

Worms are not commonly found in the mesentery or mesocolon. There are a few similar cases. In 1 case, Paragonimus westermani was located in the pelvic cavity surrounding the omentum [24]. Kim et al. [25] reported that a larva of Anisakis species was found in the lymph node within the mesocolon. In addition, Terranova type A larva was found on the serosal wall of the ileum in a patient whose clinical diagnosis was acute appendicitis [26].

Direct detection of flukes or eggs, serologic examination, and imaging findings are generally used for diagnosis of fascioliasis in humans. Initial laboratory findings showed eosino- philia in our patient. ELISA test targeting Clonorchis, Paragonimus, cysticercus, and sparganum resulted in positive reactions against cysticercus and sparganum. The patient underwent a surgical operation under the impression of cysticercosis. After the operation, ELISA test for $F$. hepatica was positive.

In fact, ELISA is important for detection of $F$. hepatica, particularly in cases involving difficult diagnosis [27]. However, in our case, cross-reaction was observed with cysticercus and sparganum. Several studies reported cross-reactions of Fasciola with other parasites $[28,29]$. However, those cross-reactions were only with Schistosoma or cestode. There is no documented evidence indicating that IgG of $F$. hepatica has cross-reactivity with the antigens of sparganum and cysticercus. Nevertheless, the possibility of cross-reaction with these parasites cannot be completely excluded. In an experimental study on cross-reactivity, Lee and Yong [30] found that the recombinant fatty acid binding protein from C. sinensis (CsFABP) cross-reacted with sera of patients with fascioliasis and paragonimiasis. This CsFABP is thought to bind with human bile acid [30].

From 1993 to 2006, in Korea, the IgG seroprevalence of clonorchiasis has been at the highest rate among cysticercosis, paragonimiasis, sparganosis, and clonorchiasis. However, because of the aforementioned cross-reactivity of clonorchiasis, it should be differentiated from other parasitic infections [31]. Use of different antigens may be required in order to preclude cross-reactions in ELISA test.

CT findings for peritoneal fascioliasis are mesenteric or omental infiltrations, lymph node enlargement, ascites, and mass or thickening of ligamentum teres [32]. Most of these lesions are located in the perihepatic area. It appears that juvenile flukes migrate from the gut mucosa to the liver parenchyma. Imaging of our case showed abscesses in the distal transverse colon and splenic flexure, which was unusual. The location of the abscess on CT images can support this possibility of direct penetration through the intestinal wall.

Histopathological examinations through surgical resection of the lesion or direct extraction of the fluke can make the confirmative diagnosis based on morphological identification. The adult worm has a flat and leaf-like appearance with a conical projection at the anterior end. The granulomatous abscess formation in the mesocolon is characteristic in fascioliasis.

Extrabiliary ectopic fascioliasis is not rare, and the related sites are quite variable. We experienced an ectopic fascioliasis case in which the adult fluke and eggs were found at the site of surgical management. Therefore, inclusion of F. hepatica 
should be considered in the differential diagnosis of parasitic infections with the help of the patient's diet history, eosinophilia, abdominal pain, and the screening ELISA test.

\section{ACKNOWLEDGMENT}

This work was supported by an Inha University Research Grant.

\section{CONFLICT OF INTEREST}

No potential conflict of interest related to this article is reported.

\section{REFERENCES}

1. Chen MG, Mott KE. Progress in assessment of morbidity due to Fasciola hepatica infection: a review of recent literature. Trop Dis Bull 1990; 87: R1-R38.

2. Mas-Coma MS, Esteban JG, Bargues MD. Epidemiology of human fascioliasis: a review and proposed new classification. Bull World Health Organ 1999; 77: 340-346.

3. Mas-Coma S. Epidemiology of fascioliasis in human endemic areas. J Helminthol 2005; 79: 207-216.

4. Curtale F, Hassanein YA, Barduagni P, Yousef MM, Wakeel AE, Hallaj Z, Mas-coma S. Human fascioliasis infection: gender differences within school-age children from endemic areas of the Nile Delta, Egypt. Trans R Soc Trop Med Hyg 2007; 101: 155-160.

5. Gonzalez LC, Esteban JG, Bargues MD, Valero MA, Ortiz P, Naquira C, Mas-Coma S. Hyperendemic human fascioliasis in Andean valleys: an altitudinal transect analysis in children of Cajamarca province, Peru. Acta Trop 2011; 120: 119-129.

6. Mera y Sierra R, Agramunt VH, Cuervo P, Mas-Coma S. Human fascioliasis in Argentina: retrospective overview, critical analysis and baseline for future research. Parasit Vectors 2011; 4: 104.

7. Lee SH, Cho SY, Seo BS, Choe KJ, Chi JG. A human case of ectopic fascioliasis in Korea. Korean J Parasitol 1982; 20: 191-200.

8. Park CI, Kim H, Ro JY, Gutierrez Y. Human ectopic fascioliasis in the cecum. Am J Surg Pathol 1984; 8: 73-77.

9. Cho SY, Seo BS, Kim YI, Won CK, Cho SK. A case of human fascioliasis in Korea. Korean J Parasitol 1976; 14: 47-152.

10. Kim YH, Kang KJ, Kwon JH. Four cases of hepatic fascioliasis mimicking cholangiocarcinoma. Korean J Hepatol 2005; 11: 169175.

11. Ha SW, Chang HH, Kim SW, Lee JM, Cho CM, Kong HH, Chung DI. A case of fascioliasis diagnosed by endoscopic nasobiliary drainage fluid examination. Korean J Med 2007; 72: 658662.

12. Makay O, Gurcu B, Caliskan C, Nart D, Tuncyurek M, Korkut M. Ectopic fascioliasis mimicking a colon tumor. World J Gastroen- terol 2007; 13: 2633-2635.

13. Ruggieri F, Correa AJ, Martinez E. Cerebral distomiasis. Case report. J Neurosurg 1967; 27: 268-271.

14. Arjona R, Riancho JA, Aguado JM, Salesa R, Conzalez-Macias J. Fascioliasis in developed countries: a review of classic and aberrant forms of the disease. Medicine (Baltimore) 1995; 74: 13-23.

15. Prociv P, Walker JC, Whitby M. Human ectopic fascioliasis in Australia: first case reports. Med J Aust 1992; 156: 349-351.

16. Vatsal DK, Kapoor S, Venkatesh V, Vatsal P, Husain N. Ectopic fascioliasis in the dorsal spine: case report. Neurosurgery 2006; 59: E706-E707.

17. Price TA, Tuazon CU, Simon GL. Fascioliasis: case reports and review. Clin Infect Dis 1993; 17: 426-430.

18. Han JK, Jang HJ, Choi BI, Kim SH, Kim TK, Won HJ, Kim YI, Cho SY. Experimental hepatobiliary fascioliasis in rabbits: radiology-pathology correlation. Invest Radiol 1999; 34: 99-108.

19. Lee OJ, Kim TH. Indirect evidence of ectopic pancreatic fascioliasis in human. J Gastroenterol Hepatol 2006; 21: 1631-1633.

20. Cho SY, Yang HN, Kong Y, Kim JC, Shin KW, Koo BS. Intraocular fascioliasis: a case report. Am J Trop Med Hyg 1994; 50: 349353.

21. Chang EC, Choi HL, Park YW, Kong Y, Cho SY. Subcutaneous fascioliasis: a case report. Korean J Parasitol 1991; 29: 403-405.

22. Kim HY, Choi IW, Kim YR, Quan JH, Ismail HA, Cha GH, Hong SJ, Lee YH. Fasciola hepatica in snails collected from water-dropwort fields using PCR. Korean J Parasitol 2014; 52: 645-652.

23. Marcos LA, Terashima A, Gotuzzo E. Update on hepatobiliary flukes: fascioliasis, opisthorchiasis and clonorchiasis. Curr Opin Infect Dis 2008; 21: 523-530.

24. Jeong WK, Kim Y, Kim YS, Park DW, Park CK, Baek HK, Park YW. Heterotropic paragonimiasis in the omentum. J Comput Assist Tomogr 2002; 26: 1019-1021.

25. Kim HJ, Park C, Cho SY. A case of extragastrointestinal anisakiasis involving a mesocolic lymph node. Korean J Parasitol 1997; 35: 63-66.

26. Seo BS, Chai JY, Lee SH, Hong ST, Seo JW, Noh SH. A human case infected by the larva of Terranova type A in Korea. Korean J Parasitol 1984; 22: 248-252.

27. Espinoza JR, Timoteo O, Herrera-Velit P. Fas2-ELISA in the detection of human infection by Fasciola hepatica. J Helminthol 2005; 79: 235-240.

28. Azab M el-s, el Zayat EA. Evaluation of purified antigens in haemagglutination test (IHA) for determination of cross reactivities in diagnosis of fascioliasis and schistosomiasis. J Egypt Soc Parasitol 1996; 26: 677-685.

29. Wuhrer M, Grimm C, Dennis RD, Idris MA, Geyer R. The parasitic trematode Fasciola hepatica exhibits mammalian-type glycolipids as well as Gal (beta1-6) Gal-terminating glycolipids that account for cestode serological cross-reactivity. Glycobiology 2004; 14: 115-126.

30. Lee JS, Yong TS. Expression and cross-species reactivity of fatty acid-binding protein of Clonorchis sinensis. Parasitol Res 2004; 93: 339-343. 
31. Lee MK, Hong SJ, Kim HR. Seroprevalence of tissue invading parasitic infections diagnosed by ELISA in Korea. J Korean Med Sci 2010; 25: 1272-1276.
32. Song KD, Lim JH, Kim MJ, Jang YJ, Kim JW, Cho SH, Kwon JH. Peritoneal manifestations of fascioliasis on CT images: a new observation. Abdom Imaging 2013; 38: 839-843. 